\title{
MRI texture analysis as a predictor of tumor recurrence or progression in patients with clinically non-functioning pituitary adenomas
}

\author{
Brandon P Galm,*, E Leonardo Martinez-Salazar²*, Brooke Swearingen³, Martin Torriani², Anne Klibanski', \\ Miriam A Bredella² and Nicholas A Tritos ${ }^{1}$ \\ ${ }^{1}$ Neuroendocrine Unit, Departments of ${ }^{2}$ Radiology, and ${ }^{3}$ Neurosurgery, Massachusetts General Hospital and Harvard \\ Medical School, Boston, Massachusetts, USA \\ Correspondence \\ should be addressed \\ to B P Galm \\ *(B P Galm and E L Martinez-Salazar contributed equally to this work) \\ Email \\ brandongalm@gmail.com
}

\begin{abstract}
Background: There are limited predictors of prognosis in patients with clinically non-functioning pituitary adenomas (NFPAs). We hypothesized that MRI texture analysis may predict tumor recurrence or progression in patients with NFPAs undergoing transsphenoidal pituitary surgery (TSS).

Objective: To characterize texture parameters on preoperative MRI examinations in patients with NFPAs in relation to prognosis.

Methods: Retrospective study of patients with NFPAs who underwent TSS at our institution between 2009 and 2010. Clinical, radiological and histopathological data were extracted from electronic medical records. MRI texture analysis was performed on coronal T1-weighted non-enhanced MR images using Image J (NIH). MRI texture parameters were used to predict tumor recurrence or progression. Both logistic regression and Cox proportional hazard analyses were conducted to adjust for potential confounders.

Results: Data on 78 patients were analyzed. On both crude and multivariable-adjusted analyses, mean, median, mode, minimum and maximum pixel intensity were associated with the risk of pituitary tumor recurrence or progression after TSS. Patients whose tumor mean pixel intensity was above the median for the population had a hazard ratio of 0.44 (95\% Cl: $0.21-0.94, P=0.034)$ for recurrence or progression in comparison with tumors below the median. Conclusions: Our data suggest that MRI texture analysis can predict the risk of tumor recurrence or progression in patients with NFPAs.
\end{abstract}

\section{Introduction}

Pituitary adenomas are common, with an estimated prevalence of $10.6 \%$ of incidental sellar lesions reported in autopsy series (1) and approximately $1 / 1200$ in community-based studies $(2,3)$. Adenomas comprise about $90 \%$ of sellar masses $(4,5)$. Clinically non-functioning pituitary adenomas (NFPAs) account for approximately $40-50 \%$ of pituitary adenomas in surgical series $(4,5)$. For those patients who require intervention, including
European Journal of Endocrinology (2018) 179, 191-198 
resection) or progress (after subtotal resection), additional therapy, including radiotherapy or a second operation, may be required (6).

There is a relative paucity of information on factors that may be helpful in reliably predicting recurrence or progression after pituitary surgery, and studies have reported mixed results $(8,9,10,11,12,13)$. Reported prognostic factors include age, gender, tumor size, tumor invasion (including involvement of the cavernous sinuses or dura) and tumor histopathology (including immunohistochemistry of pituitary hormones and assessment of proliferation markers such as Ki67 and MIB-1 index). There is a need for accurate prognostic factors that can stratify patients into high- or low-risk categories and identify those who need more intensive surveillance or therapy.

Image texture analysis is an objective approach that quantifies tissue gray-level patterns using computerassisted measurements that are independent of visual interpretation (14). This technique has been used to distinguish benign vs malignant lesions, predict response to therapy and assist in prognostication in a variety of malignances, including lung, esophageal and colorectal cancer $(15,16,17,18,19)$. However, there are no studies on MRI texture analysis in pituitary adenomas. We hypothesized that MRI texture analysis is of prognostic value in patients with NFPAs. In the present study, we aimed to determine whether MRI texture analysis can predict recurrence or progression of NFPAs after pituitary surgery.

\section{Subjects and methods}

\section{Study design}

We conducted a retrospective, observational study of patients with NFPAs who underwent their first pituitary surgery by a single neurosurgeon at the Massachusetts General Hospital (MGH) from 2009 to 2010. We identified these patients through review of databases in the Department of Neurosurgery. Follow-up data were retrieved from the time of surgery until December 2017.

\section{Study population}

We included all adult patients $\geq 18$ years of age with clinically non-functioning pituitary tumors (defined below) who had a preoperative MRI available that included a coronal T1-weighted sequence and at least
12 months of follow-up postoperatively. We excluded two patients who did not have their first pituitary surgery at MGH.

\section{Data collection}

Clinical, radiological and histopathological data were extracted from the electronic medical records by a single author. Data extracted include the following: age, sex, size of tumor (maximum diameter), presence of cavernous sinus invasion (Knosp grade 2 or higher), indication for surgery, hypopituitarism pre- and postoperatively, immunohistochemical staining for pituitary hormones and assessment of proliferation markers (Ki67 or MIB-1 index), completeness of resection (dichotomously analyzed), radiological recurrence or progression (defined below), need for repeat operation and need for radiotherapy.

\section{MRI texture analysis}

MRI texture analysis $(14,20)$ was performed by a single operator who was blinded to the clinical and outcome data. Analyses were performed on the preoperative MRI using a non-enhanced coronal T1-weighted MRI image through the largest diameter of the tumor and a region of interest (ROI) containing the entire tumor was drawn. All images were obtained using 1.5 Tesla MRI equipment. Any obvious cystic or necrotic areas were excluded from the ROI. Texture analysis was performed on a dedicated workstation using Image $(\mathrm{NIH})$. The following texture parameters were obtained: skewness (asymmetry of the pixel distribution), kurtosis (degree of peaking of the pixel distribution), modal gray value (most frequently occurring gray value within the ROI), mean pixel intensity (average gray value within the ROI), median pixel intensity (median gray value of the pixels in the ROI) and maximum and minimum pixel intensities (maximum and minimum gray values within the ROI).

\section{Study definitions}

We defined NFPAs as those without a clinically overt hypersecretion syndrome including Cushing's syndrome, acromegaly or hyperthyroidism. We included patients with an elevated prolactin level as long as hyperprolactinemia was considered to be a consequence of stalk effect. In the case of macroprolactinomas, prolactin levels are usually elevated above $250 \mu \mathrm{g} / \mathrm{L}$ (21). However, as there is some disagreement about this cut-off, we also used $94 \mu \mathrm{g} / \mathrm{L}$ as a 
cut-off (22). Only a single patient had a prolactin above this latter cut-off $(165 \mu \mathrm{g} / \mathrm{L})$. We performed a sensitivity analysis excluding this case, which did not change our results.

For histopathological data, we defined positive hormonal staining as those tumors for which many or most cells stained for that hormone, and negative staining if no cells or only some or rare cells stained positive. In three cases, the Ki67 from the initial surgery was not reported as an absolute number, but was reported as either less than $1 \%$ or as extremely low. In these cases, we used a Ki67 index of $0.02 \%$ as the lower limit of detection (23). We defined hypopituitarism as deficiency of at least one pituitary hormone. This included the presence of inappropriately low serum gonadotropins in postmenopausal women. This also included those on thyroid replacement therapy, as we were unable to distinguish primary vs secondary hypothyroidism in this retrospective study.

Tumor recurrence or progression was defined based on the impression of the radiologist and neurosurgeon. The extent of resection (gross total versus subtotal) was evaluated on the 6-week postoperative MRI. Tumor recurrence was defined as the appearance of tumor on postoperative MRI images in those who had gross total resection after initial pituitary surgery. Progression was defined as enlargement of tumor on postoperative MRI images in those who had subtotal resection after initial pituitary surgery. The timing and frequency of MRI scans were determined by the neurosurgeon, but were usually performed at 6 weeks, 6 months and 12 months, and thereafter based on MRI findings (generally every 12-24 months).

\section{Ethics approval}

Approval was obtained from the Partners Human Research Committee and any requirements for informed consent were waived as this was a retrospective study with no direct patient contact.

\section{Statistical analysis}

Statistical analysis was performed using Stata 15.1 (Stata Corp, College Station, TX, USA). Data are presented as mean (standard deviation) and median (interquartile range) values, as appropriate. All results were considered significant at an alpha level of 0.05. For image texture parameters, which were highly skewed, we performed log-transformations of the data prior to inclusion in regression models. We compared means for parametric data using the $t$-test and medians for non-parametric data using the Wilcoxon rank-sum test. We used unadjusted and multivariable-adjusted logistic and Cox regression models to determine which variables were associated with recurrence or progression. Proportionality of the hazards in Cox models was tested using Schoenfeld residuals and by including an interaction term for each variable with the logarithm of time.

\section{Results}

\section{Patient demographics}

A total of 78 cases met our inclusion criteria and were included in our analysis. Baseline characteristics of these patients are shown in Table 1. Overall, the average age was 54.2 (12.0) years and 58\% of patients were male. Patients were followed for a median of 67 (50-79) months, with a maximum follow-up time of 99 months. Most patients (69\%) had pituitary surgery because of vision loss or compression of the optic chiasm without vision loss. Most

Table 1 Baseline characteristics of included patients $(n=78)$. Data are presented as $n(\%)$ or as stated.

\begin{tabular}{l} 
Age (years), mean (s.D.) \\
Sex, male \\
Size (cm), mean (s.D.) \\
Cavernous sinus invasion \\
Hypopituitarism preoperatively \\
Hypopituitarism postoperatively \\
Follow-up time (months), median (IQR) \\
Indication for surgery \\
Vision loss \\
Headache \\
Hypopituitarism \\
Apoplexy \\
Compression of optic chiasm without vision loss \\
Other (including interval growth) \\
Immunohistochemistry staining* \\
GH positive \\
PRL positive \\
ACTH positive \\
TSH positive \\
FSH positive \\
LH positive \\
Alpha-subunit positive \\
Ki67 or MIB-1 index (\%), median (IQR) \\
Gross total resection \\
Recurrence of tumor \\
Subtotal resection \\
Progression of tumor \\
Required repeat surgery at MGH \\
Received radiotherapy at MGH \\
\hline
\end{tabular}

*Only those in which many or most tumor cells stained positive. 
tumors (89\%) stained on immunohistochemistry, with FSH-beta staining being the most common (23\%) out of all pituitary hormones. Forty-seven percent of patients had gross total resection at the time of surgery, and $8 \%$ of these subsequently recurred. On the other hand, 53\% of patients had subtotal resection, and 35\% of these tumors progressed. Only $10 \%$ of patients required repeat surgery, and $22 \%$ of all patients received radiotherapy.

\section{Predictors of recurrence or progression}

Using Cox proportional hazards regression (adjusting for completeness of resection, age and Ki67), we found that Ki67 index (crude hazard ratio (HR): 1.17, 95\% CI: 1.041.32, for each 1 unit increase, $P=0.012$; adjusted HR: 1.18 , $95 \%$ CI: $1.05-1.32, P=0.005)$ and completeness of resection (crude HR: 0.21, 95\% CI: 0.08-0.5, complete vs incomplete resection, $P<0.001$; adjusted HR: $0.21,95 \%$ CI: $0.08-0.5$, $P=0.001$ ) were strongly associated with recurrence or progression. However, we did not find crude or adjusted associations of cavernous sinus invasion, indication for surgery, size of tumor or immunohistochemical staining patterns with recurrence or progression (data not shown).

\section{MRI texture analysis}

The association of MRI texture parameters and recurrence or progression of pituitary tumors is shown in Table 2. On both crude and multivariable-adjusted analyses, mean, median, mode, minimum and maximum pixel intensities were associated with tumor recurrence or progression. Kurtosis and skewness were not associated with tumor recurrence or progression on either crude or adjusted analyses.
In order to provide a clinically relevant estimate as to the effect of MRI texture analysis, we categorized the log-transformed mean pixel intensity into those above the median and those below (Fig. 1). As compared to those below the median, those above the median had a HR of 0.44 (95\% CI: $0.21-0.94, P=0.034)$ for recurrence or progression.

We also repeated our analyses with the outcome as the composite of recurrence or progression on MRI, need for radiotherapy or need for repeat surgery. This included one extra patient who received prophylactic radiotherapy for a large residual tumor surrounding one of the cavernous carotid arteries. Including this additional case in our analysis did not change any of our results (data not shown).

\section{Histopathological characteristics}

Four patients had tumors that stained positive for $\mathrm{GH}$; there was no clinical suspicion or features of acromegaly preoperatively in any of these patients. Three out of four had normal IGF-I levels preoperatively; only one had a marginally elevated IGF-I level $(286 \mathrm{ng} / \mathrm{mL}$, reference 41-279). We did not have IGF-I data for this patient on repeat endocrine testing postoperatively.

Four patients had tumors that stained positive for $\mathrm{ACTH}$; there was no clinical suspicion or features of Cushing's syndrome in any of these patients. Two had normal 24-h urinary free cortisol levels preoperatively, and a third one had two normal late-night salivary cortisol levels preoperatively and normal ACTH stimulation testing at 2 months postoperatively. The fourth patient did not have any tests for hypercortisolism preoperatively and remains on glucocorticoid replacement 7 years after surgery.

Table 2 Association of MRI texture parameters and recurrence (after gross total resection) or progression (after subtotal resection) of pituitary tumor on follow-up MRI using Cox proportional hazards regression. Data are presented as medians (IQR) of the original, non-transformed data. $P$ values were calculated using log-transformed data for parameters that were highly skewed: mean, mode, minimum, maximum, median.

\begin{tabular}{|c|c|c|c|c|c|c|}
\hline $\begin{array}{l}\text { Image texture } \\
\text { parameter }\end{array}$ & $\begin{array}{c}\text { Recurrence or } \\
\text { progression }(n=33)\end{array}$ & $\begin{array}{c}\text { No recurrence or } \\
\text { progression }(n=45)\end{array}$ & Crude HR $^{\dagger}$ & $\begin{array}{c}\text { Crude } \\
P \text { value }\end{array}$ & Adjusted* HR $^{+}$ & $\begin{array}{c}\text { Adjusted* } \\
\text { P value }\end{array}$ \\
\hline Mean & $261(147-315)$ & $346(266-625)$ & $0.38(0.18-0.80)$ & 0.009 & $0.44(0.21-0.94)$ & 0.015 \\
\hline Median & $260(147-312)$ & $347(266-627)$ & $0.38(0.18-0.80)$ & 0.009 & $0.44(0.21-0.94)$ & 0.015 \\
\hline Mode & 257 (147-309) & 343 (259-649) & $0.38(0.18-0.78)$ & 0.010 & $0.41(0.19-0.87)$ & 0.014 \\
\hline Minimum & 166 (95-213) & $220(172-414)$ & $0.49(0.24-1.00)$ & 0.015 & $0.46(0.22-0.98)$ & 0.019 \\
\hline Maximum & $373(206-459)$ & $467(331-830)$ & $0.40(0.19-0.83)$ & 0.006 & $0.44(0.21-0.94)$ & 0.012 \\
\hline Skewness & $0.023(-0.099$ to 0.268$)$ & $-0.004(-0.178$ to 0.176$)$ & $0.87(0.44-1.75)$ & 0.906 & $0.88(0.42-1.86)$ & 0.735 \\
\hline Kurtosis & $0.386(0.148-0.81)$ & $0.325(-0.001$ to 1.009$)$ & $1.14(0.57-2.27)$ & 0.737 & $1.57(0.76-3.25)$ & 0.753 \\
\hline
\end{tabular}




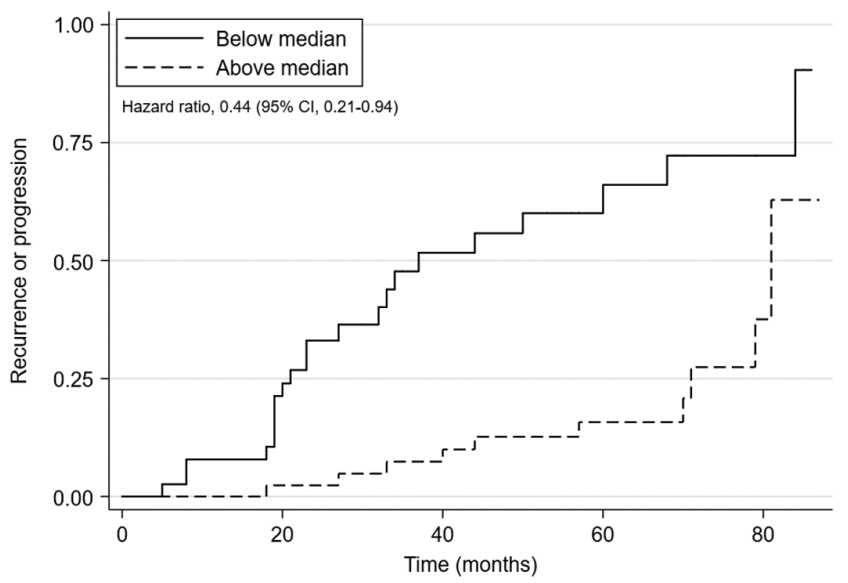

Figure 1

Adjusted Kaplan-Meier curves comparing recurrence or progression among those patients with tumors showing log-transformed mean pixel intensity above the median vs those below the median. Curves are adjusted for Ki67 index and completeness of resection. Adjusted curves for the median, mode, minimum and maximum pixel intensity are not shown but appear identical.

Because functioning adenomas, especially somatotroph and corticotroph tumors, may behave differently from NFPAs, we performed additional sensitivity analyses excluding the above eight cases. This yielded identical results on both crude and multivariableadjusted analyses (data not shown).

Sixteen patients had tumors that stained positive for prolactin. All these patients underwent surgery because of mass effect and inappropriately low prolactin level relative to tumor size. Only one of these patients had a preoperative prolactin level greater than $94 \mu \mathrm{g} / \mathrm{L}$ (prolactin $165 \mu \mathrm{g} / \mathrm{L}$ ). Excluding this patient in a sensitivity analysis did not affect the results (data not shown). When we excluded all 16 patients whose tumors stained positive for prolactin ( $21 \%$ of our sample), the results of the crude analysis remained unchanged, while the association barely missed statistical significance $(P=0.059$ for mean pixel intensity) in the adjusted model.

\section{Correlation of MRI texture analysis with other parameters}

As a secondary (exploratory) analysis, we aimed to determine whether any MRI texture parameters strongly correlated with other clinical, radiological or histopathological parameters. We did not find that any of the MRI texture parameters correlated with age, Ki67 index, sex, cavernous sinus invasion, completeness of resection or immunohistochemical staining (data not shown).

\section{Discussion}

Clinically NFPAs are common pituitary tumors that may require intervention because of mass effect, including compression of the optic chiasm. First-line therapy is typically TSS (6). However, there are limited prognostic factors that can be used to stratify patients based on their risk of recurrence or progression after pituitary surgery. Markers that have been proposed include age, sex, tumor size, tumor invasion and tumor histopathology. However, results of these studies have been mixed and inconsistent $(8,9,10,11,12,13)$. Considering that a significant proportion of tumors recur or progress, it is important to determine which patients are at higher risk so that the degree of tumor surveillance and intervention can be tailored accordingly.

In this report, we found that several MRI texture parameters, including mean, median, mode, minimum and maximum pixel intensity were strongly associated with recurrence or progression of NFPAs after surgery, both before and after adjusting for completeness of resection, Ki67 index and age. Tumors with a log-transformed mean pixel intensity above the median had a HR of 0.44 for recurrence or progression compared to those below the median. We did not find that image texture parameters were correlated with other clinical, radiological or histopathological factors.

Numerous studies have investigated the association between texture parameters and clinical outcomes such as prognosis and response of malignant tumors to therapy (20). Furthermore, several studies have also characterized the association between texture parameters and histopathological correlates of tumor heterogeneity, such as markers of hypoxia and angiogenesis, proliferation (Ki67) and tumor grade $(24,25,26)$. Heterogeneity at the tissue level is thought to be reflected in changes in texture parameters, which would signify a tumor more likely to recur or progress $(27,28)$. In this study, we did not find that Ki67 correlated with texture parameters, which is in contrast to a previous study performed in patients with non-small-cell lung cancer (NSCLC) (25). However, NSCLC is much more aggressive than pituitary adenomas, with higher Ki67 values, and this may account for the differences in results. Furthermore, we did not perform higher-order texture analyses, which accounted 
for the association in some studies; it may be that these parameters correlate with histopathological findings in pituitary adenomas.

Recent studies have investigated the association of image texture analysis with genetic and methylation defects. In squamous cell carcinomas of the head and neck, texture parameters were able to predict p53 status, an important tumor suppressor (29). Furthermore, in gliomas, texture analysis was able to predict O6-methylguanine-DNA-methyltransferase methylation status, which has also been identified (in some studies) as an important predictor of response to temozolomide in atypical pituitary adenomas (30). While we did not evaluate these markers, the differences in image texture parameters in our study may be explained by fundamentally different pathophysiological processes at the cellular level, and it would be important to explore this further in future studies.

We found that texture analysis was a strong predictor of recurrence or progression, at least initially (Fig. 1). There was a large separation of the survival curves in the first few years of follow-up; however, after 6-7 years, the difference between the survival curves appears to narrow. It may be that the tumor heterogeneity changes over time, with changes at the cellular level in residual or recurrent tumors, such that repeat texture analysis during follow-up may be helpful; however, it is also important to note that only 16 patients (21\% of our cohort) had follow-up times greater than 80 months, thus limiting our ability to make reliable conclusions about the survival curves after this time.

We believe that our findings are important for several reasons. First, there are a limited number of prognostic factors that can be used to help predict whether NFPAs will recur or progress after pituitary surgery. Second, some of the most important prognostic factors, such as completeness of resection or histopathology, can only be obtained invasively (i.e. at the time of surgery). In contrast, image texture analysis is a non-invasive technique that can be applied post hoc to preoperative imaging with minimal risk to the patient.

There are several strengths of our study. This is the first study to investigate the use of image texture analysis in pituitary adenomas. We included a cohort of patients who underwent pituitary surgery by a single experienced neurosurgeon at a single center. Our image texture analysis was performed by a single radiologist who was also blinded to clinical and outcomes data. Furthermore, our clinical outcomes are consistent with other reports in the literature; our remission rate after surgery was $47 \%$, and $8 \%$ of these tumors recurred, similar to the $47 \%$ and $10.6 \%$, respectively, found in a recent meta-analysis (7). Both silent corticotroph and silent somatotroph adenomas have been reported to be more aggressive than other NFPAs $(31,32)$. We did not find that ACTH or $\mathrm{GH}$ positivity on immunohistochemistry was associated with recurrence or progression, but we only had four patients in either category, limiting our power to detect any difference.

There are some limitations to our study. Most importantly, our study design was retrospective, and patients were followed for variable periods of time; some patients came from remote geographic areas and were not clinically followed in the long term in our clinic after surgery. However, serial postoperative MRI scans were obtained for surveillance and were reviewed in our center in these cases. While we included several image texture parameters, we did not conduct higher-order analyses, such as co-occurrence matrices and model- and transformbased methods. While there was no pre-defined protocol as to the frequency and timing of MRI examinations, all patients were followed by a single neurosurgeon, minimizing any significant differences in follow-up and management. Finally, we only used 1.5 Tesla images in our analysis, as this was the magnet strength being used routinely at our center.

We found that many of the tumor cells in our series stained positive for pituitary hormones, despite the absence of clinical or laboratory evidence of a hypersecretion syndrome. This could be attributed to silent adenomas or poorly functioning tumors, to differences in staining procedures and interpretation or to the limited sample size in our series. We found that 5\% of our tumors stained positive for ACTH, which is similar to that found in large series (5). However, we found that $21 \%$ of our tumors stained positive for prolactin (compared to $2 \%$ in the literature), 5\% stained positive for GH (compared to $\sim 1 \%$ ) and $8 \%$ stained positive for TSH (compared to $\sim 1 \%)(5)$. While we considered all of these clinically nonfunctioning, the implications of these findings in relation to image texture parameters are unclear. However, all these patients underwent surgery because of mass effect or tumor growth (rather than hormone excess), which underscores the clinical relevance of our analysis to patients with NFPAs. Furthermore, excluding cases that stained positive for ACTH or GH in a sensitivity analysis did not affect our results.

In our sensitivity analysis excluding the single patient with significant hyperprolactinemia, our results did not change. However, when we excluded all 16 patients whose 
tumors stained positive for prolactin, the association between recurrence or progression and image texture parameters was no longer significant in the multivariableadjusted model. This may be due to differences in behavior of the tumors; silent prolactinomas may be slightly more likely to recur, and perhaps may do so in a different time course, as compared to NFPAs (7). However, it is important to note that these 16 cases comprised $21 \%$ of our cohort, and thus, our power to detect any difference without these cases is significantly limited. Furthermore, as these tumors were not associated with significant hyperprolactinemia, they would likely be managed as NFPAs clinically. Thus, our findings are relevant to the management of clinical NFPAs, some of which may be poorly functioning prolactinomas.

With our findings in mind, we believe that there is substantial potential for this technique to be applied to pituitary tumors in general. We included only NFPAs undergoing pituitary surgery. Prospective confirmation of our findings would be helpful in future studies.

\section{Declaration of interest}

The authors declare that there is no conflict of interest that could be perceived as prejudicing the impartiality of this study.

\section{Funding}

This research did not receive any specific grant from any funding agency in the public, commercial or not-for-profit sector.

\section{References}

1 Molitch ME. Nonfunctioning pituitary tumors and pituitary incidentalomas. Endocrinology Metabolism Clinics of North America 200837 151-171, xi. (https://doi.org/10.1016/j.ecl.2007.10.011)

2 Daly AF, Rixhon M, Adam C, Dempegioti A, Tichomirowa MA $\&$ Beckers A. High prevalence of pituitary adenomas: a crosssectional study in the province of Liege, Belgium. Journal of Clinical Endocrinology and Metabolism 200691 4769-4775. (https://doi. org/10.1210/jc.2006-1668)

3 Fernandez A, Karavitaki N \& Wass JA. Prevalence of pituitary adenomas: a community-based, cross-sectional study in Banbury (Oxfordshire, UK). Clinical Endocrinology 201072 377-382. (https:// doi.org/10.1111/j.1365-2265.2009.03667.x)

4 Freda PU \& Post KD. Differential diagnosis of sellar masses. Endocrinology Metabolism Clinics of North America 199928 81-117, vi. (https://doi.org/10.1016/S0889-8529(05)70058-X)

5 Saeger W, Ludecke DK, Buchfelder M, Fahlbusch R, Quabbe HJ \& Petersenn S. Pathohistological classification of pituitary tumors: 10 years of experience with the German Pituitary Tumor Registry. European Journal of Endocrinology 2007156 203-216. (https://doi. org/10.1530/eje.1.02326)

6 Swearingen B. Update on pituitary surgery. Journal of Clinical Endocrinology and Metabolism 201297 1073-1081. (https://doi. org/10.1210/jc.2011-3237)

7 Roelfsema F, Biermasz NR \& Pereira AM. Clinical factors involved in the recurrence of pituitary adenomas after surgical remission: a structured review and meta-analysis. Pituitary 201215 71-83. (https://doi.org/10.1007/s11102-011-0347-7)

8 Brochier S, Galland F, Kujas M, Parker F, Gaillard S, Raftopoulos C, Young J, Alexopoulou O, Maiter D \& Chanson P. Factors predicting relapse of nonfunctioning pituitary macroadenomas after neurosurgery: a study of 142 patients. European Journal of Endocrinology 2010163 193-200. (https://doi.org/10.1530/ EJE-10-0255)

9 Comtois R, Beauregard H, Somma M, Serri O, Aris-Jilwan N $\&$ Hardy J. The clinical and endocrine outcome to transsphenoidal microsurgery of nonsecreting pituitary adenomas. Cancer 199168 860-866. (https://doi.org/10.1002/10970142(19910815)68:4<860::AID-CNCR2820680431>3.0.CO;2-4)

10 Jaffrain-Rea ML, Derome P, Bataini JP, Thomopoulos P, Bertagna X $\&$ Luton JP. Influence of radiotherapy on long-term relapse in clinically non-secreting pituitary adenomas. A retrospective study (1970-1988). European Journal of Medicine 19932 398-403.

11 Losa M, Mortini P, Barzaghi R, Ribotto P, Terreni MR, Marzoli SB, Pieralli S \& Giovanelli M. Early results of surgery in patients with nonfunctioning pituitary adenoma and analysis of the risk of tumor recurrence. Journal of Neurosurgery $2008 \mathbf{1 0 8} 525-532$. (https://doi. org/10.3171/JNS/2008/108/3/0525)

12 Meij BP, Lopes MB, Ellegala DB, Alden TD \& Laws ER Jr. The longterm significance of microscopic dural invasion in 354 patients with pituitary adenomas treated with transsphenoidal surgery. Journal of Neurosurgery 200296 195-208. (https://doi.org/10.3171/ jns.2002.96.2.0195)

13 O'Sullivan EP, Woods C, Glynn N, Behan LA, Crowley R, O'Kelly P, Smith D, Thompson CJ \& Agha A. The natural history of surgically treated but radiotherapy-naive nonfunctioning pituitary adenomas Clinical Endocrinology 200971 709-714. (https://doi.org/10.1111/ j.1365-2265.2009.03583.x)

14 Tourassi GD. Journey toward computer-aided diagnosis: role of image texture analysis. Radiology 1999213 317-320. (https://doi. org/10.1148/radiology.213.2.r99nv49317)

15 Ganeshan B, Burnand K, Young R, Chatwin C \& Miles K. Dynamic contrast-enhanced texture analysis of the liver: initial assessment in colorectal cancer. Investigative Radiology 201146 160-168. (https:// doi.org/10.1097/RLI.0b013e3181f8e8a2)

16 Ganeshan B, Panayiotou E, Burnand K, Dizdarevic S \& Miles K. Tumour heterogeneity in non-small cell lung carcinoma assessed by CT texture analysis: a potential marker of survival. European Radiology 201222 796-802. (https://doi.org/10.1007/s00330-0112319-8)

17 Ganeshan B, Skogen K, Pressney I, Coutroubis D \& Miles K. Tumour heterogeneity in oesophageal cancer assessed by CT texture analysis: preliminary evidence of an association with tumour metabolism, stage, and survival. Clinical Radiology 201267 157-164. (https://doi. org/10.1016/j.crad.2011.08.012)

18 Miles KA, Ganeshan B, Griffiths MR, Young RC \& Chatwin CR. Colorectal cancer: texture analysis of portal phase hepatic CT images as a potential marker of survival. Radiology $2009 \mathbf{2 5 0} 444-452$. (https://doi.org/10.1148/radiol.2502071879)

19 Ng F, Ganeshan B, Kozarski R, Miles KA \& Goh V. Assessment of primary colorectal cancer heterogeneity by using whole-tumor texture analysis: contrast-enhanced CT texture as a biomarker of 5-year survival. Radiology 2013266 177-184. (https://doi. org/10.1148/radiol.12120254)

20 Davnall F, Yip CS, Ljungqvist G, Selmi M, Ng F, Sanghera B, Ganeshan B, Miles KA, Cook GJ \& Goh V. Assessment of tumor heterogeneity: an emerging imaging tool for clinical practice? Insights into Imaging 20123 573-589. (https://doi.org/10.1007/ s13244-012-0196-6)

21 Melmed S, Casanueva FF, Hoffman AR, Kleinberg DL, Montori VM, Schlechte JA \& Wass JA. Diagnosis and treatment of hyperprolactinemia: an Endocrine Society clinical practice guideline. 
Journal of Clinical Endocrinology and Metabolism 201196 273-288. (https://doi.org/10.1210/jc.2010-1692)

22 Karavitaki N, Thanabalasingham G, Shore HC, Trifanescu R, Ansorge O, Meston N, Turner HE \& Wass JA. Do the limits of serum prolactin in disconnection hyperprolactinaemia need re-definition? A study of 226 patients with histologically verified non-functioning pituitary macroadenoma. Clinical Endocrinology $2006 \mathbf{6 5}$ 524-529. (https://doi.org/10.1111/j.1365-2265.2006.02627.x)

23 Thapar K, Kovacs K, Scheithauer BW, Stefaneanu L, Horvath E, Pernicone PJ, Murray D \& Laws ER Jr. Proliferative activity and invasiveness among pituitary adenomas and carcinomas: an analysis using the MIB-1 antibody. Neurosurgery 199638 99-106; discussion 106-107. (https://doi.org/10.1097/00006123-199601000-00024)

24 Ganeshan B, Goh V, Mandeville HC, Ng QS, Hoskin PJ \& Miles KA. Non-small cell lung cancer: histopathologic correlates for texture parameters at CT. Radiology 2013266 326-336. (https://doi. org/10.1148/radiol.12112428)

25 Miles KA, Ganeshan B \& Hayball MP. CT texture analysis using the filtration-histogram method: what do the measurements mean? Cancer Imaging 201313 400-406. (https://doi.org/10.1102/14707330.2013.9045)

26 Skogen K, Ganeshan B, Good C, Critchley G \& Miles K. Measurements of heterogeneity in gliomas on computed tomography relationship to tumour grade. Journal of Neuro-Oncology 2013111 213-219. (https://doi.org/10.1007/s11060-012-1010-5)

27 Ganeshan B \& Miles KA. Quantifying tumour heterogeneity with CT. Cancer Imaging 201313 140-149. (https://doi.org/10.1102/14707330.2013.0015)

28 Castellano G, Bonilha L, Li LM \& Cendes F. Texture analysis of medical images. Clinical Radiology 200459 1061-1069. (https://doi. org/10.1016/j.crad.2004.07.008)

29 Dang M, Lysack JT, Wu T, Matthews TW, Chandarana SP, Brockton NT, Bose P, Bansal G, Cheng H, Mitchell JR et al. MRI texture analysis predicts $\mathrm{p} 53$ status in head and neck squamous cell carcinoma. American Journal of Neuroradiology 201536 166-170. (https://doi.org/10.3174/ajnr.A4110)

30 Lau Q, Scheithauer B, Kovacs K, Horvath E, Syro LV \& Lloyd R. MGMT immunoexpression in aggressive pituitary adenoma and carcinoma. Pituitary 201013 367-379. (https://doi.org/10.1007/ s11102-010-0249-0)

31 Ben-Shlomo A \& Cooper O. Silent corticotroph adenomas. Pituitary 201821 183-193. (https://doi.org/10.1007/s11102-0180864-8)

32 Langlois F, Woltjer R, Cetas JS \& Fleseriu M. Silent somatotroph pituitary adenomas: an update. Pituitary 201821 194-202. (https:// doi.org/10.1007/s11102-017-0858-y)

Received 9 April 2018

Revised version received 7 June 2018

Accepted 3 July 2018 